\title{
Glaucoma triple procedure of extracapsular cataract extraction, posterior chamber lens implantation, and trabeculectomy
}

\author{
S P B PERCIVAL \\ From the Department of Ophthalmology, Scarborough District Hospital, Scarborough, Yorkshire
}

SUMmaRY Thirty-four consecutive glaucoma triple procedures with a minimum follow up of six months have been shown to be effective and safe. Satisfactory control of the glaucoma was achieved in each case, and $91 \%$ of eyes were returned to an acuity of $6 / 12$ or better. $1 \%$ sodium hyaluronate was found to be beneficial by preventing scleral or corneal collapse during surgery and ensuring an even filtration postoperatively without hypotony.

Glaucoma and cataract are often separated as surgical entities, for one requires a wound that leaks and the other a wound completely watertight. However, their frequent coexistence has led to a wealth of ideas as to how best to control the glaucoma and at the same time improve the vision. Many successful designs have been made for the operations to be combined, ${ }^{\prime}$ but occasional over filtration may be associated with shallow anterior chamber, choroidal detachment, and peripheral anterior synechia, so that the introduction of simultaneous lens implantation could prove an unnecessary hazard. The consensus from lens implant surgeons is that chronic glaucoma is a relative contraindication to lens implantation, and that when it is indicated the glaucoma should be controlled prior to cataract surgery." Some authorities believe this should be the approach even when lens implantation is not under consideration, provided the patient can withstand two procedures, and particularly as cataract surgery alone may prove more complicated in patients with glaucoma than in those without. ${ }^{7}$ It is also known that intracapsular cataract extraction (ICCE) may lead to failure of a previously successful filtration procedure, $2 \times "$ and that unoperated glaucoma can become more difficult to control in the aphakic eye. $1 "$ "I Extracapsular cataract extraction (ECCE) may prove difficult because of a bound down or poorly dilating pupil. However, when ECCE is chosen a later trabeculectomy is likely to be as successful as if it were performed on a phakic eye, and there are no

Correspondence to Mr S P B Percival, FRCS. problems of vitreous possibly blocking the filtration site or adhering to the wound. Theoretically, therefore, when a combined procedure is considered trabeculectomy should have a higher incidence of success when combined with extracapsular than with intracapsular extraction.

$1 \%$ sodium hyaluronate (Healonid) may contribute to the effectiveness of the combined procedure by preventing scleral or corneal collapse during surgery and by ensuring an even filtration postoperatively without anterior chamber shallowing.

So with these points under consideration this communication presents an improved single procedure for the treatment of cataract with glaucoma.

\section{Material and methods}

Thirty-four consecutive glaucoma triple procedures have been analysed with a minimum follow up of six months and a maximum follow up of four years (mean 14 months). All patients received trabeculectomy combined with ECCE. Six received a Boberg Ans posterior chamber implant, and 28 received a Sinskey or Pearce flexible loop posterior chamber implant. All eyes had intraocular pressures persistently above $21 \mathrm{mmHg}$ before treatment, and all eyes had visual acuity reduced to less than $6 / 24$ because of cataract. The 34 eyes of 33 patients were divided into the following three groups: (1) eight cases of ocular hypertension or preglaucoma (OHT) showing no field defect and a cup disc ratio of less than $0 \cdot 65$; (2) 21 cases of chronic simple (open-angle) glaucoma (CSG) with a field defect in the operated or 
fellow eye and a cup disc ratio of 0.65 or greater in the operated eye; (3) five cases of chronic angle closure glaucoma (CACG).

Two eyes from group (2) and four from group (3) had received previous glaucoma surgery. Two had had previous filtration procedures, and four had had previous peripheral iridectomies, including one eye that had also received a subsequent trabeculectomy. All eyes that had suffered previous glaucoma surgery had small bound down pupils from formation of posterior synechiae. There were no cases of secondary glaucoma.

Table 1 shows the age range, the mean intraocular pressures at presentation, and the mean intraocular pressures prior to surgery after various combinations of pilocarpine, timolol, neutral adrenaline and guanethidine. Despite this topical therapy 16 eyes had pressures persistently above $21 \mathrm{mmHg}$.

The glaucoma triple procedure comprised: (1) A fornix based conjunctival flap. (2) A two-thirds depth scleral incision $5 \mathrm{~mm}$ of which was placed $4 \mathrm{~mm}$ posterior to the limbus for the trabeculectomy, $7 \mathrm{~mm}$ placed $1 \mathrm{~mm}$ posterior to the limbus for later insertion of the implant. (3) The outer trabeculectomy flap was dissected into the cornea and extended the entire length of the incision so that the part not used for trabeculectomy formed a two-plane step. (4) The base incision for the trabeculectomy $2.5 \mathrm{~mm}$ posterior to the limbus. (5) A $1 \mathrm{~mm}$ stab incision and anterior capsulotomy under sodium hyaluronate. (6) Completion of the trabeculectomy. In those eyes with bound down pupils stage 6 was done before stage 5 with conversion of peripheral iridectomy to broad (sector) iridotomy and inferior sphincterotomy, in order to facilitate the capsulotomy. Sodium hyaluro-

Table 1 Age and preoperative intraocular pressure

\begin{tabular}{|c|c|c|c|c|c|c|}
\hline & \multirow[t]{2}{*}{ Eyes } & \multirow[t]{2}{*}{$\begin{array}{l}\text { Age } \\
\text { range (yr) }\end{array}$} & \multicolumn{2}{|c|}{$\begin{array}{l}\text { Presenting } \\
I O P(m m H g)\end{array}$} & \multicolumn{2}{|c|}{$\begin{array}{l}\text { Preoperative } \\
I O P(\min H g)\end{array}$} \\
\hline & & & Mean & Range & Mean & Range \\
\hline (1) $\mathrm{OHT}$ & 8 & $6(1)-80$ & 26 & $23-30$ & 21 & $17-26$ \\
\hline (2) CSG & 21 & $65-84$ & 30 & $23-56$ & 22 & $16-28$ \\
\hline (3) $\mathrm{CACG}$ & 5 & $66-79$ & 42 & $26-60$ & 25 & $20)-32$ \\
\hline
\end{tabular}

nate was used not only to protect intraocular tissues from inadvertent trauma but also to prevent scleral or anterior chamber collapse during surgery. (7) Completion of the $12 \mathrm{~mm}$ step incision. (8) Removal of a disc of anterior capsule with Kelman McPhearson forceps through a medium of sodium hyaluronate. (9) Injection of sodium hyaluronate behind the lens nucleus and extraction of the nucleus by scleral depression without counterpressure or reflection of the cornea. (10) Non-automated aspiration/irrigation of residual lens cortex after placement of a central direct suture. (11) Closure of the trabeculectomy flap to one side of the direct suture. (12) Intraocular lens insertion to the other side of the direct suture through a medium of sodium hyaluronate without reflecting the cornea. (13) Wound closure with three further direct scleral sutures of $10 / 0$ nylon. (14) Injection of sodium hyaluronate under trabeculectomy flap. (15) Securing of the apron of conjunctiva with a limbal $8 / 0$ Vicryl suture at each end of the wound and buried knot. (16) Subconjunctival injection of DepoMedrone (methylprednisolone acetate) with Soframycin (framycetin sulphate) and topical application of timolol maleate.

\section{Results}

Table 2 shows the movement of postoperative intraocular pressure. The majority of eyes underwent applanation tonometry at eight hours, all at 22 hours. Six $(21 \%)$ of the cases in groups 1 or 2 were found to have pressures raised above $30 \mathrm{mmHg}$ during the first 24 hours, but by the third day there were no high pressures. By six months all pressures in these two groups were below $20 \mathrm{mmHg}$ though for three eyes timolol drops had had to be restarted, and there was a net mean fall of $6.1 \mathrm{mmHg}$ since surgery. Despite several cases of moderately severe optic atrophy 26 of the 29 eyes were seeing $6 / 9$ or better. The other three eyes suffered from macular degeneration.

The five cases of CACG were expected to prove more difficult to treat. Intraocular pressures were all uncontrolled despite treatment, and four had bound down pupils from previous surgery. However, all had a satisfactory response to treatment with total control of intraocular pressure. All were seeing at six months

Table 2 Postoperative intraocular pressure $(\mathrm{mmHg})$

\begin{tabular}{|c|c|c|c|c|c|c|c|}
\hline & \multicolumn{2}{|c|}{ First 24 hours } & \multicolumn{2}{|c|}{ Third day } & \multicolumn{2}{|c|}{ Six months } & \multirow{2}{*}{$\begin{array}{l}\text { Mean fall from } \\
\text { preoperative } \\
\text { level }\end{array}$} \\
\hline & Mean & Range & Mean & Range & Mean & Range & \\
\hline (1) $\mathrm{OHT}$ & 17 & $7-32$ & 15 & $12-20$ & 14 & $12-16$ & $6.3 \mathrm{mmHg}$ \\
\hline (2) CSG & $24 \cdot 5$ & $5-46$ & $15 \cdot 9$ & $11-25$ & $15 \cdot 8$ & $12-19^{*}$ & $6.0 \mathrm{mml} / \mathrm{g}$ \\
\hline (3) CACG & 15 & $12-18$ & $15 \cdot 4$ & $12-23$ & 14 & $12-16$ & $11.0 \mathrm{mmHg}$ \\
\hline
\end{tabular}

*Three eyes required additional timolol drops. 
$6 / 12$ or better and showed a mean fall in intraocular pressure of $11 \mathrm{mmHg}$.

I.ONG TERM FOLLOW UP

Thirteen eyes have been followed up for more than two years and four for more than three. All these eyes at the final point of follow up were seeing $6 / 12$ or better, though one had required a posterior capsulotomy to achieve this. The intraocular pressure has been maintained at below $19 \mathrm{mmHg}$ in all, 11 requiring no additional medication and two requiring additional timolol drops. Of the other 21 eyes one required a capsulotomy at six months because of capsule fibrosis, and three eyes had received primary capsulotomy. Thus 29 eyes have so far maintained a clear posterior capsule. It should be expected that some of these will require capsulotomy at a future date, but this is not a difficult procedure even with a posterior lens in situ. The posterior capsule is not therefore regarded as a problem but rather as an aid to surgery, as it ensures stability of the implant and of the posterior segment of the eye:

\section{COMPLICATIONS}

There were no cases of overfiltration with ocular hypotony, choroidal detachment, or shallowing of the anterior chamber. Apart from minor surgical hyphaemas there was one eye with secondary hyphaema that lasted four weeks but recovered $6 / 9$ vision. Three lens implants were found to decentre upwards toward the trabeculectomy site but not far enough to trouble the patient with a crescent of aphakic vision. There were no cases of implant subluxation or postoperative corneal oedema.

Three eyes developed pupil trap (or creep). Starting with adherence of the pupil margin to the posterior capsule the pupil was found slowly to creep behind the lens implant as already described in glaucoma eyes by Rock and Rylander.'" Two eyes showed 'against the rule' astigmatism higher than $+2.5 \mathrm{D}$, of $+4.0 \mathrm{D}$ and $+5.0 \mathrm{D}$ respectively, with the axis corresponding to bleb formation at the trabeculectomy site.

\section{Discussion}

The above complications signify an increased morbidity after this operation compared with standard extracapsular surgery, possibly owing to the unidirectional flow of aqueous and an increased tendency to low grade uveitis. But this is outweighed by the advantages of combining the operations into one procedure, and there were no cases of visual loss in the short term resulting from a complication of surgery. The low grade uveitis signified by mild ciliary injection with minimal cellular activity in the anterior chamber persisting in a few eyes for up to eight weeks was not associated with any failure of filtration. Neither was the presence of the implant found in any way to compromise the filtration bleb or the resulting visual acuity.

The absence so far of any of our cases resulting in inadequate glaucoma control is encouraging and, in agreement with others, ${ }^{+10} 11$ suggests a preference for trabeculectomy rather than cyclodialysis during the combined procedure. Galin and Baras ${ }^{13}$ showed that after ICCE and cyclodialysis $40 \%$ of patients required additional medication, and that in $10 \%$ of eyes the glaucoma remained uncontrolled. In Montgomery and Gills's ${ }^{x}$ large series of ECCE/cyclodialysis/ posterior chamber implant triples $38 \%$ of patients required additional glaucoma medication before one year had passed, and 13\% remained uncontrolled, but of those that were controlled there was no attenuation with time over a three-year period. $3 \%$ of patients had required surgical reformation for flat anterior chamber in the early postoperative period.

The incidence of shallow or flat anterior chamber after the combined procedure of intracapsular extraction and trabeculectomy, though absent in Rich's series, ${ }^{+}$has been variously reported by others as being between $7 \%$ and $16 \% .^{7101+15}$ This complication seems to be avoided by placement of Healonid (sodium hyaluronate, sodium chloride, sodium phosphate dihydrate, sodium acid phosphate monohydrate) in the anterior chamber and under the trabeculectomy flap to ensure an even filtration in the early postoperative period. The manoeuvre appeared to be successful in preventing a shallow chamber or any complication from ocular hypotony. However, six eyes sustained a high intraocular pressure during the first 24 hours after operation. This is a phenomenon already experienced without the use of Healonid, +11 is and to prevent compromise of the optic nerve it is important to guard against it and to continue timolol drops for at least two days after surgery.

The choice of ECCE over ICCE during the combined procedure seems to confer the following advantages: (1) theoretically better control of the glaucoma, since the eye behaves like a phakic eye, and there will be no possibility of vitreous adhering to the filtration site; further, if a second filtration procedure were to become necessary at a future date, for the same reasons, this would be less hazardous after ECCE than after ICCE; (2) the facility to insert a purely posterior chamber implant without the need for polypropylene loops anteriorly or iris fixation sutures as would be necessary after ICCE; (3) better visual results as has been suggested before. ${ }^{16}$ The fact that $91 \%$ of these eyes had a postoperative acuity of $6 / 12$ or better is partly explained by the absence of 
eyes with near absolute glaucoma. However, consideration must also be given to the fact that the presence of an intact lens capsule and absence of hypotony both contribute to a reduction of macular oedema. Other authors using the ICCE technique give a postoperative $6 / 12$ acuity incidence varying between $42 \%$ and $66 \% .+57 \% 101+15$ Clearly there must be considerable variation in any given series over the health of individual optic nerves, but an already compromised nerve cannot be helped by even mild (and often undiagnosed) macular oedema. It is significant that Montgomery and Gills, "who used the ECCE technique, alone gave a $6 / 12$ or better incidence at one year of over $80 \%$.

The single problem presented by ECCE concerned the bound down or poorly dilating pupils. These eyes were managed with radial iridotomies and inferior sphincterotomies but without secondary iris suturing, which was considered a possibly hazardous extra manipulation. A possible disadvantage here is a crescent of aphakic vision in those eyes with a wide palpebral aperture. However, among these particular patients there were no complaints of diplopia or pupillary abnormality.

\section{CONCLUSION}

The results suggest that with the facility of sodium hyaluronate this glaucoma triple procedure is both safe and effective, and that the inclusion of a posterior chamber implant does not jeopardise either the glaucoma control or the visual improvement.

\section{References}

1 Harrington DO. Cataract and glaucoma. Management of the cocxistent conditions and description of a new operation combining lens extraction with reverse cyclodialysis. A m J Ophthalmol 1966: 61: 1134-8.

2 Galin MA. Baras I, Sambursky J. Glaucoma and cataract. A study of cyclodialysis-Iens extraction. Am J Ophthalmol 1969; 67: 522-7.

3 Witmer R. Kombinierte Glaukom-Katarakt operation. Ophthalmologica 1972: 165: 203-5.

4 Rich W. Cataract extraction with trabeculectomy. Trans Ophthalmol Soc UK 1974; 94: 458-67.

5 Eustace P. Harun AOSM. Trabeculectomy combined with cataract extraction. Trans Ophthalmol Soc UK 1974; 94: 1058-63.

6 Richardson KT. Glaucoma and pscudophakia. In: Rosen ES, Haining WH, Arnott EJ, cds. Intraocular lens implantation. St Louis: Mosby, 1984: 619-21.

7 Spacth GL. Management of patients with conjoint cataract and glaucoma. Ophthalmic Surg 1980); 11: 78()-3.

8 Montgomery D. Gills JP. Extracapsular extraction, Iens implantation and cyclodialysis. Ophthalmic Surg 1980; 11:343-7.

9 Shiclds MB. Combined cataract extraction and glaucoma surgery. Ophthalmology 1982; 89: 231-7.

10) Johns GE, Layden WE. Combined trabeculectomy and cataract extraction. Am J Ophthalmol 1979; 88: 973-81.

11 Witmer R, Rohen JW. Combined cataract glaucoma operation. Trans Ophthalmol Soc UK 1976; 96: 256-61.

12 Rock RL, Rylander HG. Spontancous iris retraction after extracapsular extraction and posterior lens implantation in patients with glaucoma. Am Intraocular Implant Soc J 1983; 9: 45-7.

13 Galin MA. Baras I. Combined cyclodialysis cataract extraction: a revicw. Ann Ophthalmol 1975; 7: 721-9.

14 Edwards RS. Trabeculectomy combined with cataract extraction: a follow up study. Br J Ophthalmol 1980; 64: 72()-4.

15 Jerndal $T$. Lundstrom $M$. Trabeculectomy combined with cataract extraction. Am J Ophthalmol 1976; 81: 227-31.

16 Percival SPB, Anand V. Das SK. Prevalence of aphakic retinal detachment. BrJ Ophthalinol 1981; 67: 43-5. 